\title{
Wearable Passive E-Textile UHF RFID Tag Based on a Slotted Patch Antenna with Sewn Ground and Microchip Interconnections
}

\author{
Johanna Virkki, ${ }^{1}$ Zhigang Wei, ${ }^{2}$ Aruhan Liu, ${ }^{2}$ Leena Ukkonen, ${ }^{1}$ and Toni Björninen' \\ ${ }^{1}$ Department of Electronics and Communications Engineering, Tampere University of Technology, Tampere, Finland \\ ${ }^{2}$ Department of Electronic Engineering, City University of Hong Kong, Hong Kong \\ Correspondence should be addressed to Johanna Virkki; johanna.virkki@tut.fi
}

Received 21 September 2016; Accepted 22 January 2017; Published 13 February 2017

Academic Editor: Ming-Chun Tang

Copyright (c) 2017 Johanna Virkki et al. This is an open access article distributed under the Creative Commons Attribution License, which permits unrestricted use, distribution, and reproduction in any medium, provided the original work is properly cited.

We present a wearable passive UHF RFID tag based on a slotted patch antenna comprising only textile materials (e-textile, textile substrate, and conductive yearn). As a novel manufacturing approach, we realize the patch-to-ground and antenna-to-IC interfaces using only conductive thread and a sewing machine. We outline the electromagnetic optimization of the antenna for body-worn operation through simulations and present a performance comparison between the e-textile tag and a tag produced using regular electronics materials and methods. The measured results show that the textile tag achieves the electrical performance required in practical applications and that the slotted patch type antenna provides stable electromagnetic performance in different body-worn configurations.

\section{Introduction}

Wireless body area networks (WBAN) are revolutionizing healthcare and medicine [1-4]. Passive UHF (ultra-high frequency) RFID (radiofrequency identification) inspired technology has been identified as a compelling approach to achieve energy efficient and versatile wireless technology for future WBANs [5-8]. Here, the wireless communication of digital data by means of modulated scattering offers a unique way to implement antenna-sensors and remotely powered and programmable platforms, where an ultra-low-power microsystem integrated with an antenna provides identification and sensing functionalities [9-12]. In the current technology, UHF RFID tags are remotely addressable at distances from several meters beyond 20 meters in specialized applications. This offers a great potential for identification, sensing, and monitoring.

In WBANs, the wearable passive UHF RFID technology is envisioned to enable remote monitoring of movement and physiological parameters in assisted living and bedside applications in hospitals and nursery homes [13-17], for instance. In body-centric systems, the fundamental challenge in the antenna development raises from the proximity of the human body: the dielectric biological matter exhibits notable electrical conductivity and polarizability. This leads to reduction in the antennas' radiation performance through the consumption of energy in the interaction between the antenna's electromagnetic (EM) fields and the body. This inherently limits the attainable radiation efficiency of especially single-layered antennas [18]. In comparison, multilayered structures, such as microstrip patch [19], (planar) inverted-F antennas [20$22]$, and antennas based on substrate-integrated waveguides and metamaterials [23-25], benefit from the built-in antennabody isolation provided by the ground plane. The interaction through the fringing fields at the edges of the antenna can be further alleviated through a strategic extension of the ground plane [26].

Wearable applications require antennas and electronic devices to be embedded into clothing. Generally they need to be small, lightweight, conformal, and reliable. A widely studied approach to meet these requirements is fabrication of antennas from electrically conductive textiles (e-textiles) [23, 27-29]. They are compatible with regular textile processing, which enables the seamless integration of electronics 


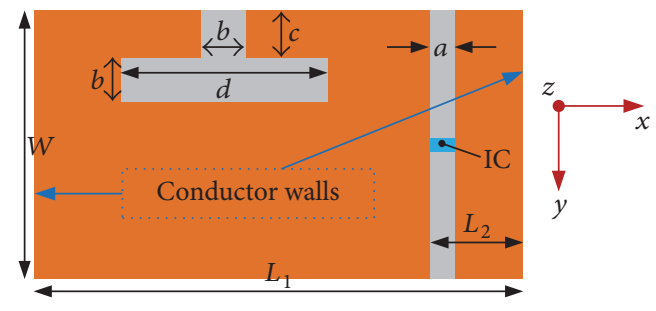

FIgURE 1: Structural diagram of the slotted patch antenna. Dimensions of the antenna in (mm): $L_{1}: 64.4, L_{2}: 13.6, W: 33.5, a: 2, b: 5, c$ : 4.75, $d: 31.1$.

in clothing. Such unnoticeable technology is desirable for comfort and aesthetic reasons, but also in patient monitoring in, for example, dementia care, where the patients may seek to remove unfamiliar devices. However, another challenge lies in the manufacturing of the required interconnections by methods that are compatible with regular textile processing. Embroidery with conductive yarn is a simple fabrication method that has been used in wearable antenna fabrication on various fabric materials [30-33]. In addition, sewing is a promising method for embedding electrical interconnections in textile materials [34-37].

In this work, we show that the conductor walls acting as the interconnections between the radiating patch and ground plane in a slotted patch antenna are readily and reliably formed by sewing with conductive yarn. In addition, we present an embroidered interconnection between the etextile and an RFID microchip fixture. We also outline the process of the EM optimization of the antenna in the bodyworn configuration and explore its platform-tolerance by measuring the on-body performance in different parts of the human body.

\section{Tag Development and Simulation Results}

The antenna development was initiated by considering the slotted patch antenna structure in Figure 1. It originates from [38], where the structure was proposed to miniaturize a twoshorted patch antenna to achieve a small metal-mountable passive UHF RFID tag that remains well-functioning on a conductive surface. Antennas for wearable tags share the same requirement of platform-tolerance: the tag must operate well near the human body, where the biological matter exhibits notable conductivity and polarizability. For this reason, we considered the slotted patch antenna a promising candidate for the development of wearable tags as well.

However, a regular circuit board process is not a viable approach to wearable devices. To achieve lightweight and conformal structure, which is compatible with textile manufacturing, we patterned the antenna conductor from nickel- and copper-plated Less EMF Shieldit Super Fabric (Cat. \#A1220) and used EPDM (Ethylene-Propylene-DieneMonomer) cell rubber foam (thickness of $4 \mathrm{~mm}$ ) as the substrate. The electrotextile has a sheet resistance of approximately $0.16 \Omega / \square$ and the dielectric constant and loss tangent of EPDM are 1.26 and 0.007 , respectively, at $915 \mathrm{MHz}$.

Optimization of the antenna structure was based on EM modelling in ANSYS High Frequency Structure Simulator
(HFSS) with the target of maximal tag read range at $915 \mathrm{MHz}$. To account for the influence of the human body on the EM properties of the antenna, we placed the antenna on a large rectangular block (thickness: $6 \mathrm{~cm}$ ) of a dielectric material with the dielectric properties of the human skin. The dielectric properties of skin were modelled using the frequencydependent four-term Cole-Cole dispersion model provided in [39], which gives the dielectric constant and loss tangent of 41.33 and 0.414 , respectively, for skin at $915 \mathrm{MHz}$. Since skin is the tissue type closest to the antenna and has dielectric properties similar to, for example, muscle, which is commonly a subsequent deeper tissue type, we considered this simplified model sufficient for our application. By using this geometrically simplified body model, we were able to estimate the antenna impedance and radiation efficiency in the proximity of the body, as well as its radiation properties when mounted on relatively large flat area of the body, such as the upper back.

Normally, the read range of a passive tag is limited by the forward link operation, that is, the efficiency of the wireless power transfer from the reader to the tag IC. Assuming free-space conditions for site-independent comparison, the obtainable tag read range at the spatial observation angles $\phi$ and $\theta$ of a spherical coordinate system centered at the tag is given by

$$
\begin{aligned}
d_{\mathrm{tag}}(\phi, \theta) & =\frac{\lambda}{4 \pi} \sqrt{\frac{\tau e_{r} D(\phi, \theta) \mathrm{EIRP}}{P_{\mathrm{ic} 0}}}, \\
\tau & =\frac{4 \operatorname{Re}\left(Z_{A}\right) \operatorname{Re}\left(Z_{\mathrm{IC}}\right)}{\left|Z_{A}+Z_{\mathrm{IC}}\right|^{2}},
\end{aligned}
$$

where $\lambda$ is the wavelength of the carrier tone emitted by the reader, EIRP is the regulated equivalent isotropic radiated power, $P_{\text {ic } 0}$ is the wake-up power of the tag IC, $e_{r}$ is the tag antenna radiation efficiency, $D$ is the tag antenna directivity, and $\tau$ is the antenna-IC power transfer efficiency determined by the antenna and IC impedances $Z_{A}$ and $Z_{\mathrm{IC}}$, respectively. Equation (1a) is a direct implication of Friis' simple transmission equation and simple circuit analysis where a generator with the internal impedance of $Z_{A}$ is connected to a load impedance of $Z_{\text {IC }}$ yields (1b) which is a measure of goodness of complex-conjugate impedance matching $(0<\tau \leq 1)$. In the simulations, the IC impedance was obtained from an equivalent parallel $\mathrm{RC}$ circuit $(R=2.85 \mathrm{k} \Omega, C=0.91 \mathrm{pF})$ developed in [40].

Compared with [38], in the present work, the operation environment of the antenna as well as the substrate thickness and material was different. Hence, we needed to reoptimize the antenna. In this process, we followed the parametric tuning guidelines in [38] to maximize $d_{\text {tag }}$ at $915 \mathrm{MHz}$ in the body-worn configuration with the help of the parametric studies in HFSS. Firstly, due to the lower permittivity of the substrate used in this work, we needed to increase the overall size of the antenna defined by parameters $W$ and $L_{1}$ slightly to lower the fundamental resonance frequency of the antenna. Likewise, we found the optimum size of the slot defined by parameters $b, c$, and $d$, to be somewhat larger than in the earlier work. The dimensions of the optimized antenna are given in Figure 1. 

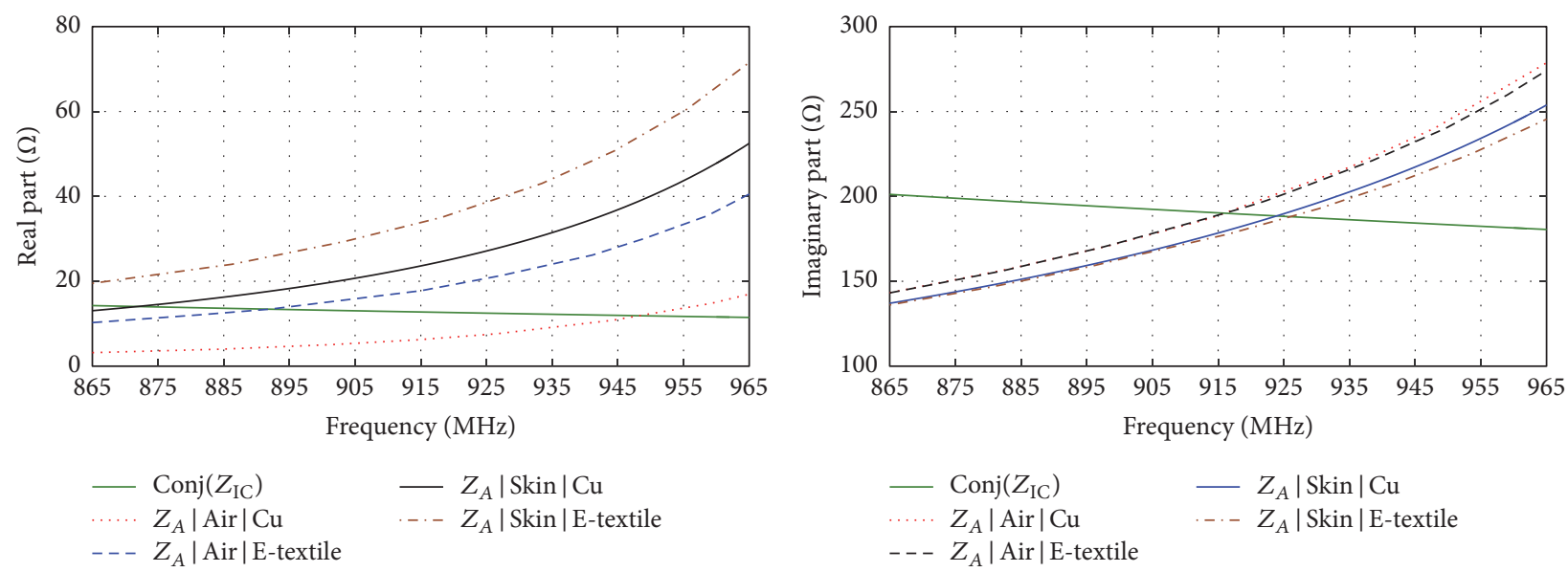

FIGURE 2: Simulated antenna impedance and the conjugate of the microchip impedance from the parallel RC equivalent circuit model $(R=$ $2.85 \mathrm{k} \Omega, C=0.91 \mathrm{pF})$.

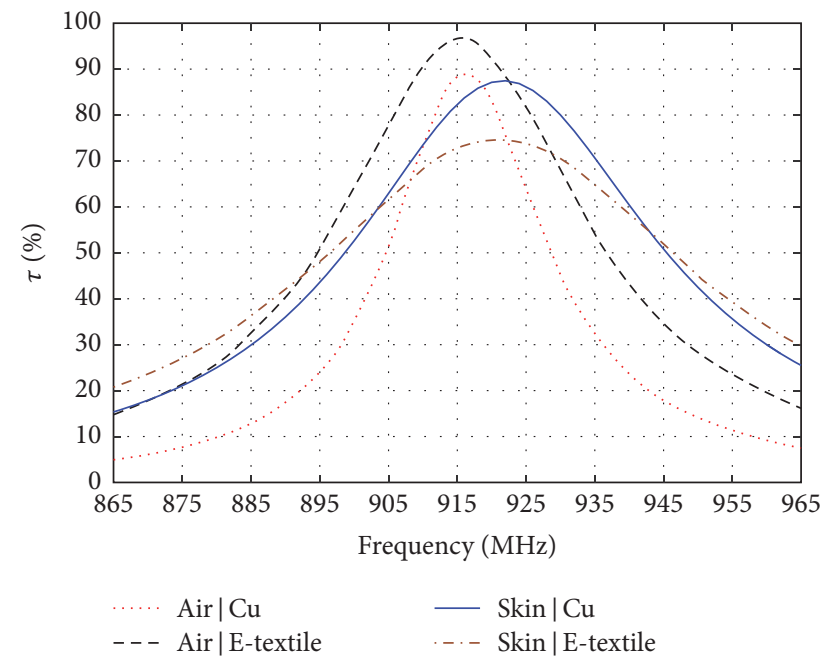

FIgURE 3: Simulated antenna-microchip power transfer efficiency.

Figure 2 compares the impedance of the antenna made of copper tape and e-textile both in air and in bodyworn configuration. Here also the complex conjugate of the microchip impedance is shown to illustrate the realization of the complex-conjugate impedance matching between the antenna and the IC. In comparison with the copper tag in air, both the presence of the body and usage of the etextile conductor manifested themselves as higher antenna resistance. This corresponds with the dissipation of energy in these materials. In contrast, the conductor material caused only minor alteration in the antenna reactance. Hence, the antenna shows good platform-tolerance in terms of impedance matching as also evidenced from Figure 3: there is little variation in the matched frequency among all the studied cases. The material loss, however, increased the impedance bandwidth.

Finally, Table 1 summarizes the radiation properties of the antenna and Figure 4 presents the simulated $d_{\text {tag }}$. It can be observed that the frequency of $d_{\text {tag }}$ correlates strongly

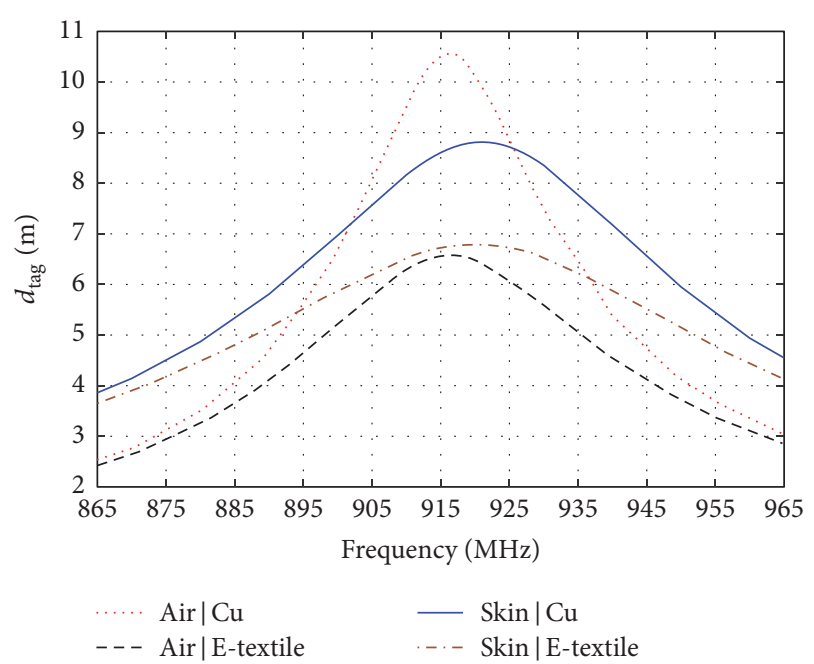

FIGURE 4: Simulated attainable read range of the tag under the emission limit of EIRP $=3.28 \mathrm{~W}$ and $P_{\mathrm{ic} 0}=-18 \mathrm{dBm}$.

with the frequency of peak $\tau$. This is because the antenna directivity $D$ and radiation efficiency $e_{r}$ (and consequently the gain $G$ ) remain relatively constant showing only a minor increasing trend with frequency seen from the results in Table 1. Another observation drawn from the data in Table 1 is that the presence of the body induces a notable increase in the antenna directivity. This helps to partly compensate the efficiency-drop due to the presence of the body. The mechanism was found to be more pronounced for the etextile antenna.

\section{Measurement Results and Discussion}

We manufactured both copper tape and e-textile versions of the tag for testing. In case of the copper tag, the antenna shape was patterned from the copper tape as a single piece using scissors and then folded over the edges of the EPDM substrate to form the whole antenna structure including the 
TABLE 1: Simulated radiation properties of the antenna.

\begin{tabular}{lccccccccc}
\hline & \multicolumn{3}{c}{$D(\mathrm{dBi})$} & \multicolumn{3}{c}{$e_{r}$} & \multicolumn{2}{c}{$G=e_{r} \cdot D(\mathrm{dBi})$} \\
\hline Freq. (MHz) & 865 & 915 & 965 & 865 & 915 & 965 & 865 & 915 \\
Air | Cu & 1.56 & 1.64 & 1.79 & 0.52 & 0.56 & 0.61 & -0.86 & -0.56 \\
Air | E-textile & 1.60 & 1.67 & 1.95 & 0.16 & 0.20 & 0.25 & -5.95 & -5.00 \\
Skin | Cu & 5.59 & 5.36 & 4.90 & 0.17 & 0.18 & 0.20 & -2.11 & -1.95 \\
Skin | E-textile & 5.61 & 5.38 & 4.89 & 0.11 & 0.13 & 0.14 & -3.87 & -3.56 \\
\hline
\end{tabular}

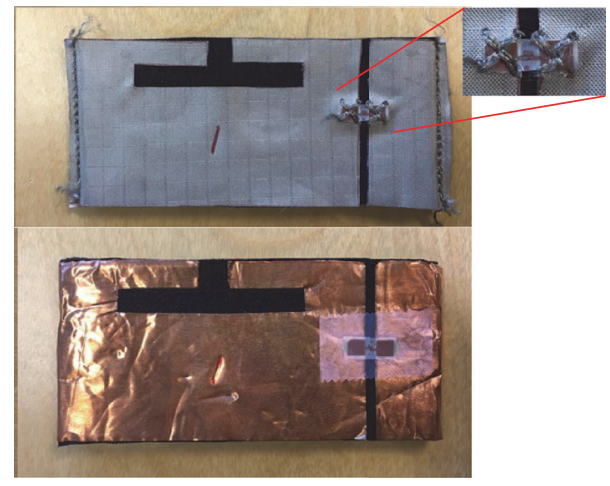

Figure 5: Samples of the manufactured e-textile (top) and copper tape (bottom) tags.

conductor walls. The RFID microchip (NXP UCODE G2iL; $P_{\text {ic } 0}=-18 \mathrm{dBm}$ ) was mounted by the manufacturer in a fixture with $3 \times 3 \mathrm{~mm}^{2}$ contact pads (copper on a plastic film) to be attached to the antenna terminals. In the copper tag, the pads were attached to the antenna using conductive epoxy (Circuit Works CW2400). The e-textile tag was, on the other hand, manufactured considering approaches, which are compatible with regular textile processing and applicable directly on larger pieces of base fabric serving as a substrate, where the conductor walls cannot be formed by folding the e-textile over the substrate edges. Thus, we started by patterning the radiating patch in two pieces, separated by the feed gap, defined by parameter $a$ in Figure 1. We then joined the two pieces by sewing a cross-shaped pattern through the microchip fixture pads and the e-textile using conductive yarn (Shieldex multifilament silver plated thread 110f34 dtex 2-ply HC) to form both mechanical attachment of the fixture and electrical connection with the IC. The e-textile we used had a hot-melt glue deposited in the backside and thus we attached the patch and ground plane to the EPDM substrate by ironing. Finally, we created the conductor walls by sewing the patch and the ground plane together with the conductive yarn. Samples of the manufactured tags are shown in Figure 5.

The fully assembled tags were tested wirelessly using Voyantic Tagformance measurement system. It contains an RFID reader with an adjustable transmission frequency $(0.8, \ldots, 1 \mathrm{GHz})$ and output power (up to $30 \mathrm{dBm}$ ) and provides the recording of the backscattered signal strength (down to $-80 \mathrm{dBm}$ ) from the tag under test. During the test, we recorded the lowest continuous-wave transmission power (threshold power: $P_{\text {th }}$ ). Here we defined $P_{\text {th }}$ as the lowest power at which a valid 16-bit random number from the tag is received as a response to the query command in ISO
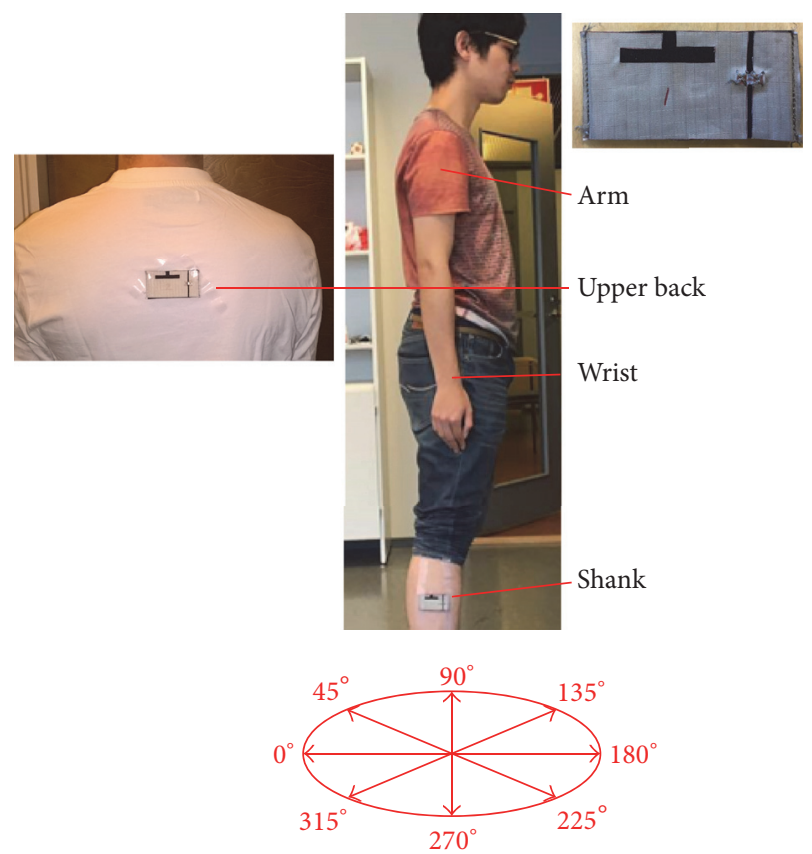

Figure 6: Measurement setup used in testing of the tags in bodyworn configurations.

18000-6C communication standard. In addition, the wireless channel from the reader antenna to the location of the tag under test was characterized using a system reference tag with known properties. As explained with details in [41] this enabled us to estimate the attainable read range of the tag $\left(d_{\text {tag }}\right)$ versus frequency from

$$
d_{\mathrm{tag}}=\frac{\lambda}{4 \pi} \sqrt{\frac{\operatorname{EIRP}}{\Lambda} \frac{P_{\mathrm{th}^{*}}}{P_{\mathrm{th}}}}
$$

where $d_{\text {tag }}$ is the measured threshold power of the tag, $\Lambda$ is a known constant describing the sensitivity of the system reference tag, $P_{\mathrm{th}^{*}}$ is the measured threshold power of the system reference tag, and EIRP is the emission limit of an RFID reader given as equivalent isotropic radiated power. Based on the calibration data provided by the manufacturer of the measurement system, we have estimated that the combined static uncertainty in $d_{\text {tag }}$ due to variability in the system reference tag $(\Lambda)$ and the output power meter of the reader $\left(P_{\mathrm{th}}, P_{\mathrm{th}^{*}}\right)$ is less than $5 \%$ throughout the studied frequency range. To evaluate the body-worn performance of the tag comprehensively, the measurements were conducted in several configurations: with tag attached to the upper back, wrist, shank, and arm of a male test subject, as indicated in Figure 6. 


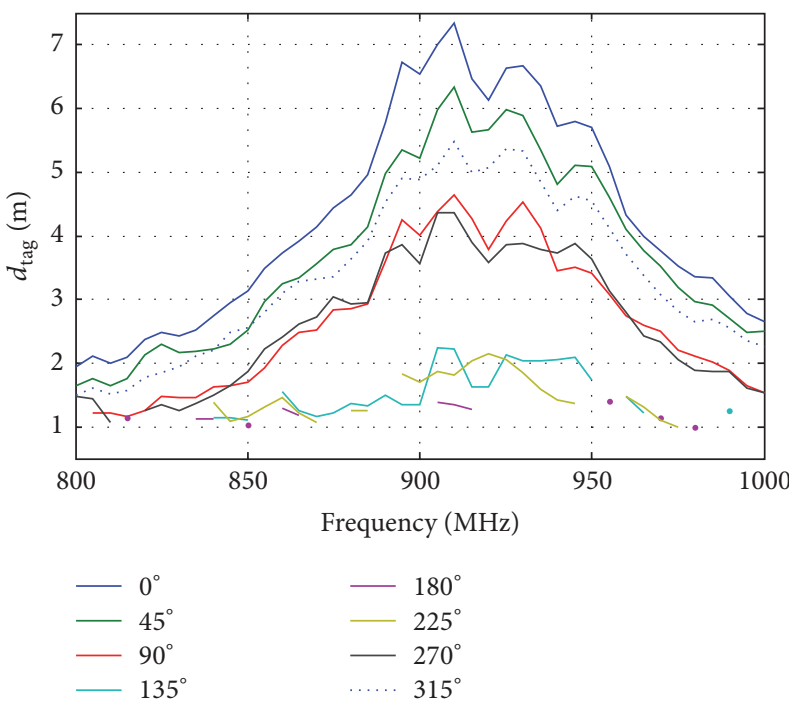

(a)
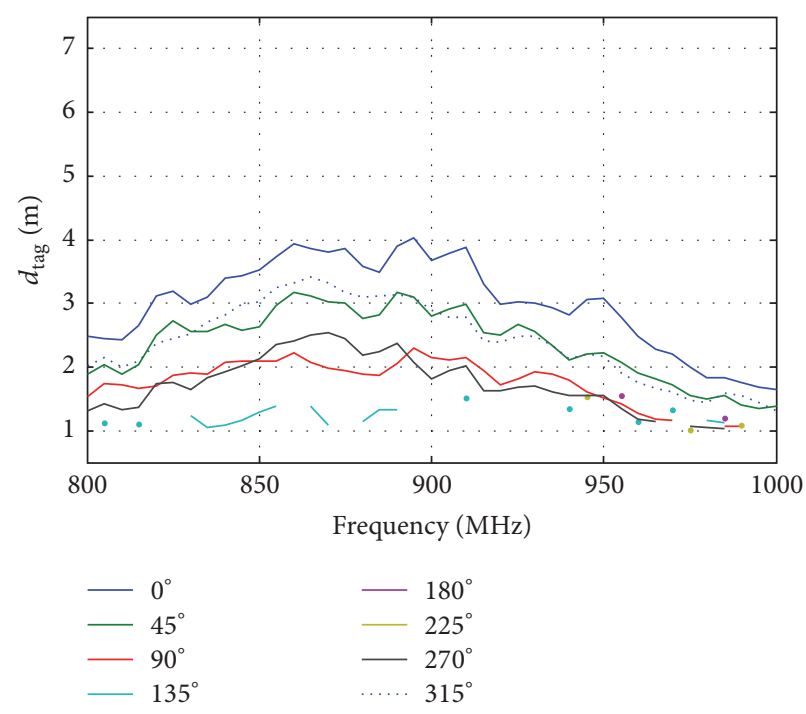

(b)

FIGURE 7: Measured attainable read range of the copper tape (a) and e-textile (b) tags attached to upper back.

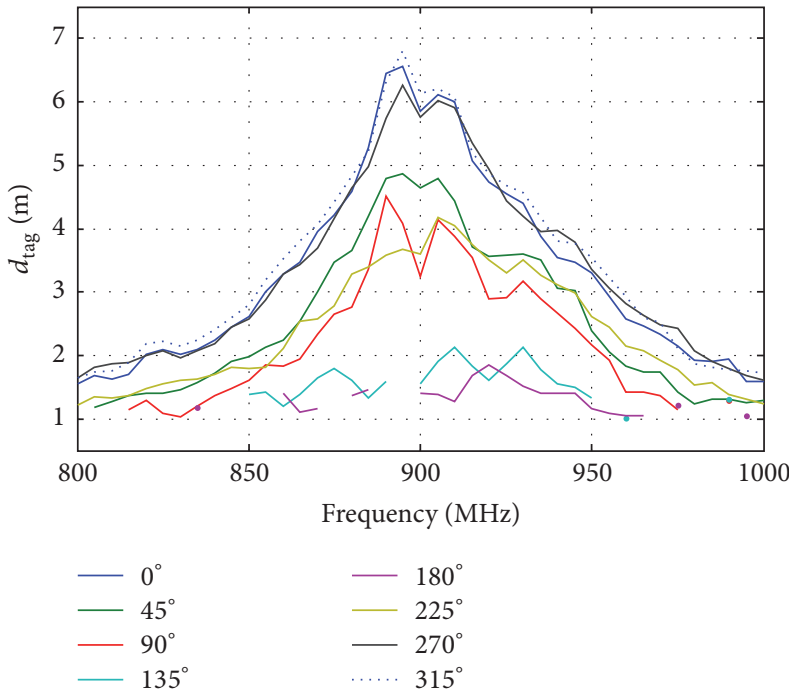

(a)

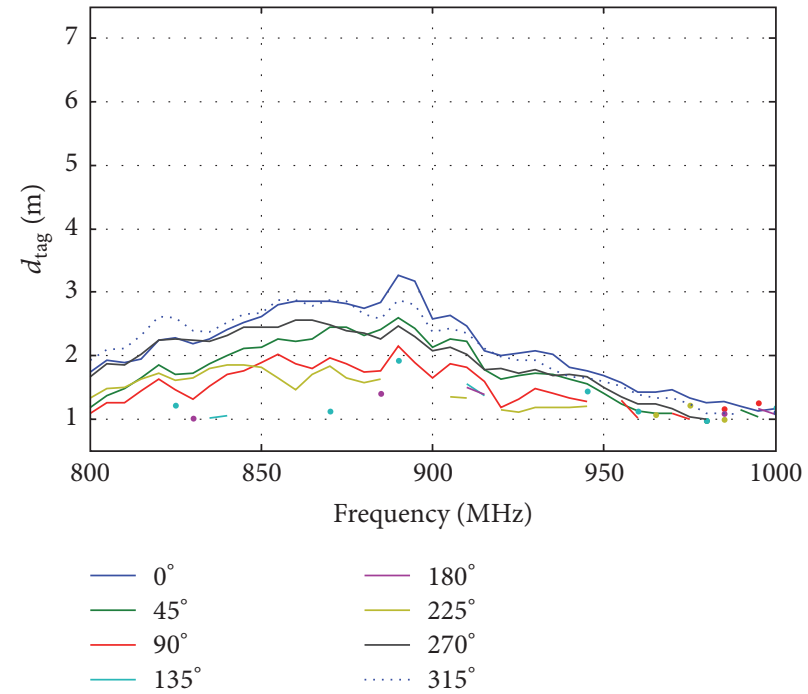

(b)

FIGURE 8: Measured attainable read range of the copper tape (a) and e-textile (b) tags attached to arm.

The corresponding estimates of the attainable read ranges of the tags are presented in Figures 7-9.

When worn in the upper back, the copper and e-textile tags achieved peak $d_{\text {tag }}$ of 7 and 4 meters, respectively, toward the line of sight (LOS) direction of $0^{\circ}$ (Figure 7). For the etextile tag, the second highest $d_{\text {tag }}$ of 3 meters was recorded from directions of $45^{\circ}$ and $315^{\circ}$. This is true for the copper tag as well, which maintained $d_{\text {tag }}$ higher than 5 meters in these directions, but due to dynamic measurement uncertainty, $d_{\text {tag }}$ recorded from the direction of $45^{\circ}$ was higher. Finally, the copper and e-textile tags remained readable also from the directions of $90^{\circ}$ and $270^{\circ}$ (i.e., within a $180^{\circ}$ beam centered at the LOS direction) with the respective $d_{\text {tag }}$ of
4 and 2 meters. In comparison with the simulation results presented in Figure 4, the measured $d_{\text {tag }}$ for the copper tag in the LOS direction was around 1.8 meters lower than the simulation. This is likely due to the difference between the actual directivity of the body-worn antenna and the estimate obtained from the geometrically simplified simulation model. However, the operation frequency was predicted correctly by the simulation. In case of the e-textile tag, the level difference between the measurement and simulation was somewhat larger. This is explained by the fact that the simulation model treated sewn interconnections as ideal.

Overall, the copper and e-textile tags showed peak $d_{\text {tag }}$ of $6, \ldots, 7$ meters and $3, \ldots, 4$ meters, respectively, when worn in arm, wrist, and shank. In arm (Figure 8), the copper 


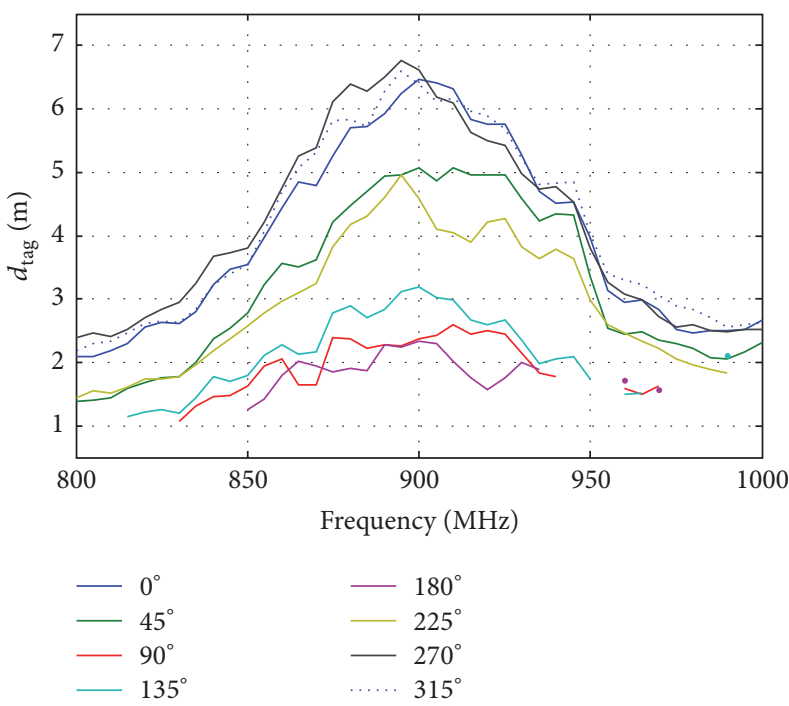

(a)
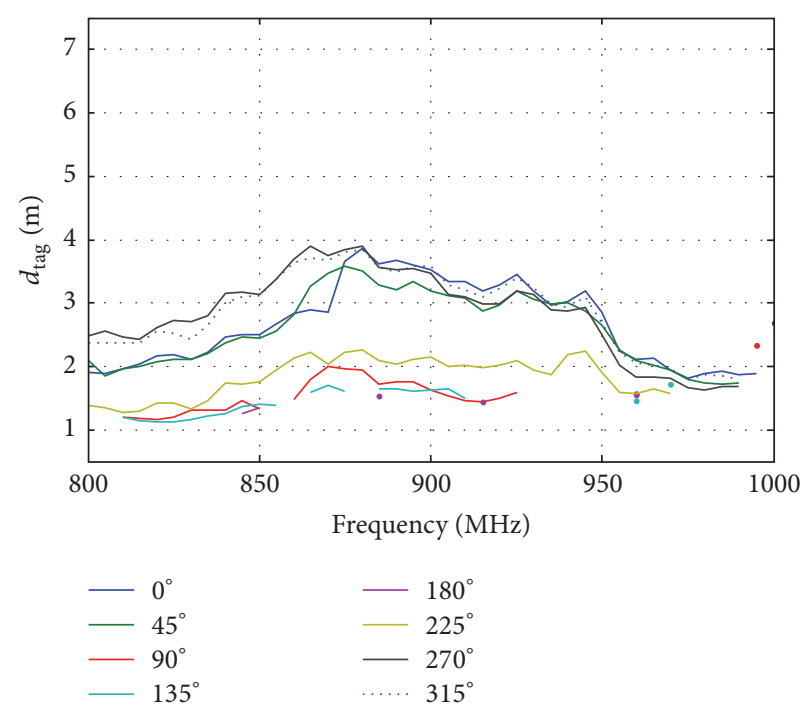

(b)

FIGURE 9: Measured attainable read range of the copper tape (a) and e-textile (b) tags attached to shank.

tag maintained peak $d_{\text {tag }}$ above of 4 meters in a $180^{\circ}$ beam through directions $225^{\circ}, 270^{\circ}, 315^{\circ}, 0^{\circ}$, and $45^{\circ}$ and the etextile tag maintained peak $d_{\text {tag }}$ above of 2 meters in a $135^{\circ}$ beam through directions $270^{\circ}, 315^{\circ}, 0^{\circ}$, and $45^{\circ}$. In comparison with the tags worn in the back, here the direction of peak $d_{\text {tag }}$ did not coincide with the LOS direction of $270^{\circ}$ but was tilted toward direction $315^{\circ}$. This can be explained by the asymmetry of the body with respect to the LOS direction. Overall the measured values were also slightly lower, which suggests that when worn in the back, the larger flat body surface backing the antenna may have induced slightly higher directivity for the antenna.

Tags worn in the shank (Figure 9) showed spatial coverage, which was similar to the case of the arm. However, here the peak $d_{\text {tag }}$ was generally higher. The beam where the e-textile tag maintained peak $d_{\text {tag }}$ above 2 meters increased from $135^{\circ}$ to $180^{\circ}$ (directions through $225^{\circ}, 270^{\circ}, 315^{\circ}, 0^{\circ}$, and $45^{\circ}$ ). Notably, shank was the only case where the copper tag maintained peak $d_{\text {tag }}$ above 2 meters from all directions. This is likely because the legs have smaller extent compared with the torso so that the diffracted beams covered better also the shadow directions.

Finally, when worn in the wrist, the conclusions drawn from the case of the arm hold with the difference that here the direction of peak $d_{\text {tag }}$ was tilted toward $225^{\circ}$ from the LOS direction and, as a result, it was the direction of $45^{\circ}$ that was limiting the 4-meter and 2-meter spatial read range coverage of the copper and e-textile tags, respectively.

\section{Conclusion}

We demonstrated the manufacturing of an e-textile passive UHF RFID tag based on a slotted patch antenna where both patch-to-ground and antenna-to-microchip interconnections were created with a sewing machine and metal plated sewing thread. In this way, wearable tags and other devices including textile-electronics interfaces can be embedded into clothing by means of regular textile processing. The measured results showed that a regular electronics manufacturing approach based on a copper conductor and the proposed textile manufacturing approach yielded attainable tag read ranges of 6 to 7 meters and 3 to 4 meters, respectively, in various common body-worn configurations. This shows that the studied tag exhibits stable body-worn performance and that the textile manufacturing approach delivers the electrical performance required in practical applications.

\section{Competing Interests}

The authors declare that there is no conflict of interests regarding the publication of this paper.

\section{Acknowledgments}

This research work was supported by the European Commission AdvIOT IRSES project, Academy of Finland, Jane and Aatos Erkko Foundation, and TEKES.

\section{References}

[1] P. Van Daele, I. Moerman, and P. Demeester, "Wireless body area networks: status and opportunities," in Proceedings of the 31st General Assembly and Scientific Symposium of the International Union of Radio Science (URSI GASS '14), Beijing, China, August 2014.

[2] Y.-L. Zheng, X.-R. Ding, C. C. Y. Poon et al., "Unobtrusive sensing and wearable devices for health informatics," IEEE Transactions on Biomedical Engineering, vol. 61, no. 5, pp. 15381554, 2014.

[3] S. Movassaghi, M. Abolhasan, J. Lipman, D. Smith, and A. Jamalipour, "Wireless body area networks: a survey," IEEE 
Communications Surveys and Tutorials, vol. 16, no. 3, pp. 16581686, 2014.

[4] H.-J. Yoo, "Your heart on your sleeve: advances in textile-based electronics are weaving computers right into the clothes we wear," IEEE Solid-State Circuits Magazine, vol. 5, no. 1, pp. 5970, 2013.

[5] A. Sani, M. Rajab, R. Foster, and Y. Hao, "Antennas and propagation of implanted RFIDs for pervasive healthcare applications," Proceedings of the IEEE, vol. 98, no. 9, pp. 1648-1655, 2010.

[6] A. Yakovlev, S. Kim, and A. Poon, "Implantable biomedical devices: wireless powering and communication," IEEE Communications Magazine, vol. 50, no. 4, pp. 152-159, 2012.

[7] J. Grosinger, "Feasibility of backscatter RFID systems on the human body," Eurasip Journal on Embedded Systems, vol. 2013, article 2, 2013.

[8] S. Rao, N. Llombart, E. Moradi et al., "Miniature implantable and wearable on-body antennas: towards the new era of wireless body-centric systems," IEEE Antennas and Propagation Magazine, vol. 56, no. 1, pp. 271-291, 2014.

[9] A. P. Sample, D. J. Yeager, P. S. Powledge, A. V. Mamishev, and J. R. Smith, "Design of an RFID-based battery-free programmable sensing platform," IEEE Transactions on Instrumentation and Measurement, vol. 57, no. 11, pp. 2608-2615, 2008.

[10] G. Marrocco, "Pervasive electromagnetics: sensing paradigms by passive RFID technology," IEEE Wireless Communications, vol. 17, no. 6, pp. 10-17, 2010.

[11] P. Pursula, J. Flak, N. Pesonen, and O. Saarela, "Intermodulation communication principle: passive wireless sensor networks for internet-of-things," in Proceedings of the 17th International Conference on Electromagnetics in Advanced Applications (ICEAA '15), pp. 1012-1015, IEEE, Torino, Italy, September 2015.

[12] M. M. Islam, K. Rasilainen, and V. Viikari, "Implementation of sensor RFID: carrying sensor information in the modulation frequency," IEEE Transactions on Microwave Theory and Techniques, vol. 63, no. 8, pp. 2672-2681, 2015.

[13] A. Dementyev and J. R. Smith, "A wearable UHF RFIDbased EEG system," in Proceedings of the IEEE International Conference on RFID (RFID '13), pp. 1-7, Orlando, Fla, USA, May 2013.

[14] R. L. S. Torres, D. C. Ranasinghe, Q. Shi, and A. P. Sample, "Sensor enabled wearable RFID technology for mitigating the risk of falls near beds," in Proceedings of the IEEE International Conference on RFID (RFID '13), pp. 191-198, Orlando, Fla, USA, May 2013.

[15] T. Kaufmann, D. C. Ranasinghe, M. Zhou, and C. Fumeaux, "Wearable quarter-wave folded microstrip antenna for passive UHF RFID applications," International Journal of Antennas and Propagation, vol. 2013, Article ID 129839, 11 pages, 2013.

[16] O. O. Rakibet, C. V. Rumens, J. C. Batchelor, and S. J. Holder, "Epidermal passive RFID strain sensor for assisted technologies," IEEE Antennas and Wireless Propagation Letters, vol. 13, pp. 814-817, 2014.

[17] S. Amendola, G. Bovesecchi, P. Coppa, and G. Marrocco, "Thermal characterization of epidermal RFID sensor for skin temperature measurements," in Proceedings of the IEEE International Symposium on Antennas and Propagation (APSURSI '16), pp. 461-462, IEEE, Fajardo, Puerto Rico, USA, June 2016.

[18] R. M. Mäkinen and T. Kellomäki, "Body effects on thin single-layer slot, self-complementary, and wire antennas," IEEE Transactions on Antennas and Propagation, vol. 62, no. 1, pp. 385-392, 2014.
[19] P. B. Samal, P. J. Soh, and G. A. E. Vandenbosch, "UWB alltextile antenna with full ground plane for off-body WBAN communications," IEEE Transactions on Antennas and Propagation, vol. 62, no. 1, pp. 102-108, 2014.

[20] S. J. Chen, T. Kaufmann, and C. Fumeaux, "Shorting strategies for a wearable L-slot planar inverted-F antenna," in Proceedings of the International Workshop on Antenna Technology: Small Antennas, Novel EM Structures and Materials, and Applications (iWAT '14), pp. 18-21, Sydney, Australia, March 2014.

[21] W. El Hajj, C. Person, and J. Wiart, "A novel investigation of a broadband integrated inverted-F antenna design; application for wearable antenna," IEEE Transactions on Antennas and Propagation, vol. 62, no. 7, pp. 3843-3846, 2014.

[22] P. J. Soh, G. A. E. Vandenbosch, S. L. Ooi, and N. H. M. Rais, "Design of a broadband all-textile slotted PIFA," IEEE Transactions on Antennas and Propagation, vol. 60, no. 1, pp. 379-384, 2012.

[23] S. Zhu and R. Langley, "Dual-band wearable textile antenna on an EBG substrate," IEEE Transactions on Antennas and Propagation, vol. 57, no. 4, pp. 926-935, 2009.

[24] H. R. Raad, A. I. Abbosh, H. M. Al-Rizzo, and D. G. Rucker, "Flexible and compact AMC based antenna for telemedicine applications," IEEE Transactions on Antennas and Propagation, vol. 61, no. 2, pp. 524-531, 2013.

[25] S. Agneessens, S. Lemey, T. Vervust, and H. Rogier, "Wearable, small, and robust: the circular quarter-mode textile antenna," IEEE Antennas and Wireless Propagation Letters, vol. 14, pp. 1482-1485, 2015.

[26] G. A. Casula, A. Michel, P. Nepa, G. Montisci, and G. Mazzarella, "Robustness of wearable UHF-band PIFAs to humanbody proximity," IEEE Transactions on Antennas and Propagation, vol. 64, no. 5, pp. 2050-2055, 2016.

[27] P. Salonen and H. Hurme, "A novel fabric WLAN antenna for wearable applications," in Proceedings of the IEEE International Antennas and Propagation Symposium and USNC/CNC/URSI North American Radio Science Meeting, pp. 700-703, June 2003.

[28] J. C. G. Matthews and G. Pettitt, "Development of flexible, wearable antennas," in Proceedings of the 3rd European Conference on Antennas and Propagation (EuCAP '09), pp. 273-277, March 2009.

[29] T. F. Kennedy, P. W. Fink, A. W. Chu, N. J. Champagne II, G. Y. Lin, and M. A. Khayat, "Body-worn E-textile antennas: the good, the low-mass, and the conformal," IEEE Transactions on Antennas and Propagation, vol. 57, no. 4, pp. 910-918, 2009.

[30] Y. Kim, K. Lee, Y. Kim, and Y. Chung, "Wearable UHF RFID tag antenna design using flexible electro-thread and textile," in Proceedings of the IEEE Antennas and Propagation Society International Symposium (AP-S '07), pp. 5487-5490, Honolulu, Hawaii, USA, June 2007.

[31] J. Choi, Y. Kim, K. Lee, and Y. Chung, "Various wearable embroidery RFID tag antenna using electro-thread," in Proceedings of the IEEE Antennas and Propagation Society International Symposium (APSURSI '08), IEEE, July 2008.

[32] G. Kim, J. Lee, K. H. Lee et al., "Design of a UHF RFID fiber tag antenna with electric-thread using a sewing machine," in Proceedings of the Asia Pacific Microwave Conference (APMC '08), Hong Kong, China, December 2008.

[33] A. Tsolis, W. G. Whittow, A. A. Alexandridis, and J. Y. C. Vardaxoglou, "Embroidery and related manufacturing techniques for wearable antennas: challenges and opportunities," Electronics, vol. 3, no. 2, pp. 314-338, 2014. 
[34] E. R. Post, M. Orth, R. R. Russo, and N. Gershenfeld, "Ebroîdery: design and fabrication of textile-based computing," IBM Systems Journal, vol. 39, no. 3-4, pp. 840-860, 2000.

[35] T. Linz, C. Kallmayer, R. Aschenbrenner, and H. Reichl, "Embroidering electrical interconnects with conductive yarn for the integration of flexible electronic modules into fabric," in Proceedings of the 9th IEEE International Symposium on Wearable Computers (ISWC '05), October 2005.

[36] T. Linz, R. Vieroth, C. Dils et al., "Embroidered interconnections and encapsulation for electronics in textiles for wearable electronics applications," Advances in Science and Technology, vol. 60, pp. 85-94, 2008.

[37] M. E. Berglund, J. Duvall, C. Simon, and L. E. Dunne, "Surfacemount component attachment for E-textiles," in Proceedings of the 19th ACM International Symposium on Wearable Computers (ISWC '15), pp. 65-66, Osaka, Japan, September 2015.

[38] T. Björninen and F. Yang, "Signal strength readout and miniaturised antenna for metal-mountable UHF RFID threshold temperature sensor tag," Electronics Letters, vol. 51, no. 22, pp. 1734-1736, 2015.

[39] S. Gabriel, R. W. Lau, and C. Gabriel, “The dielectric properties of biological tissues: III. Parametric models for the dielectric spectrum of tissues," Physics in Medicine and Biology, vol. 41, no. 11, pp. 2271-2293, 1996.

[40] T. Björninen, L. Sydänheimo, and L. Ukkonen, "Development and validation of an equivalent circuit model for UHF RFID IC based on wireless tag measurements," in Proceedings of the Antenna Measurement Techniques Association Symposium, 6 pages, Bellevue, Wash, USA, October 2012.

[41] J. Virkki, T. Björninen, S. Merilampi, L. Sydänheimo, and L. Ukkonen, "The effects of recurrent stretching on the performance of electro-textile and screen-printed ultra-highfrequency radio-frequency identification tags," Textile Research Journal, vol. 85, no. 3, pp. 294-301, 2015. 


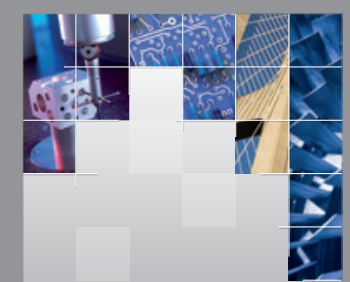

\section{Enfincering}
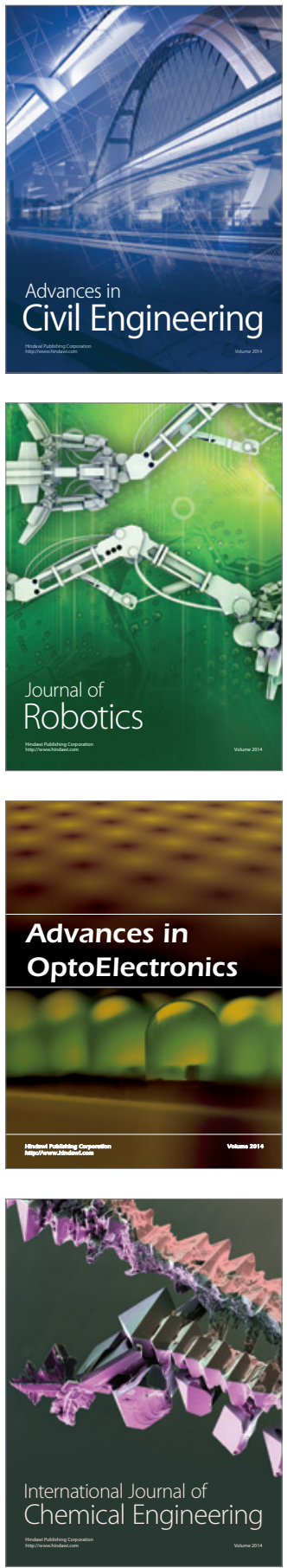

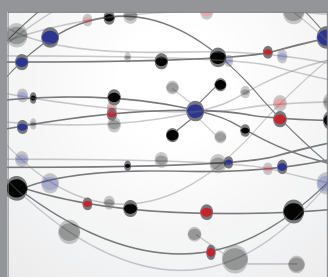

The Scientific World Journal

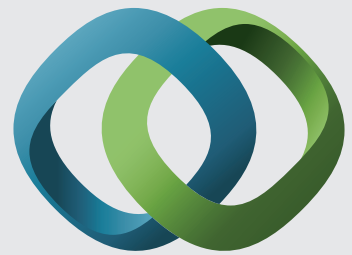

\section{Hindawi}

Submit your manuscripts at

https://www.hindawi.com
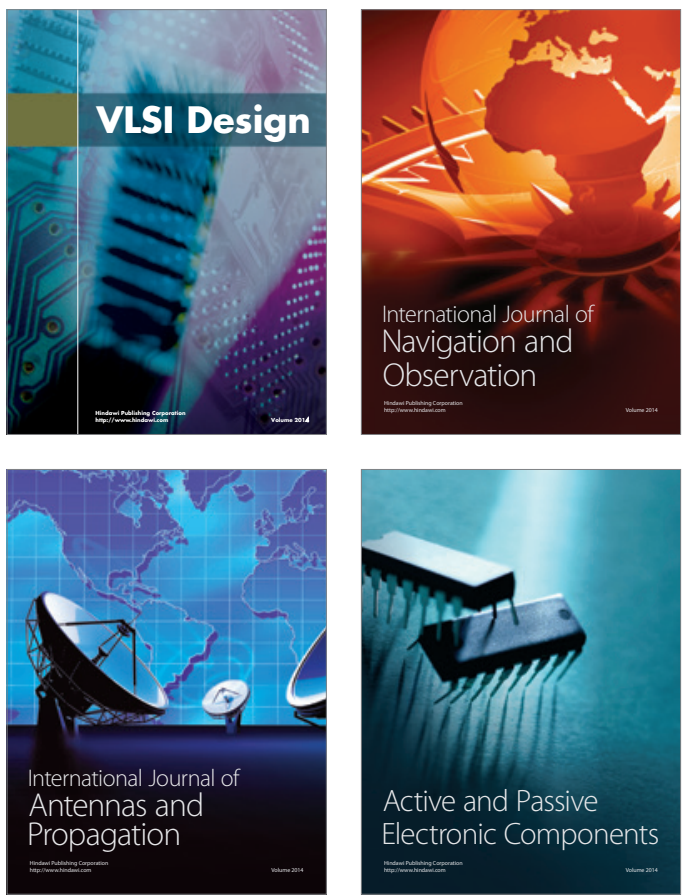
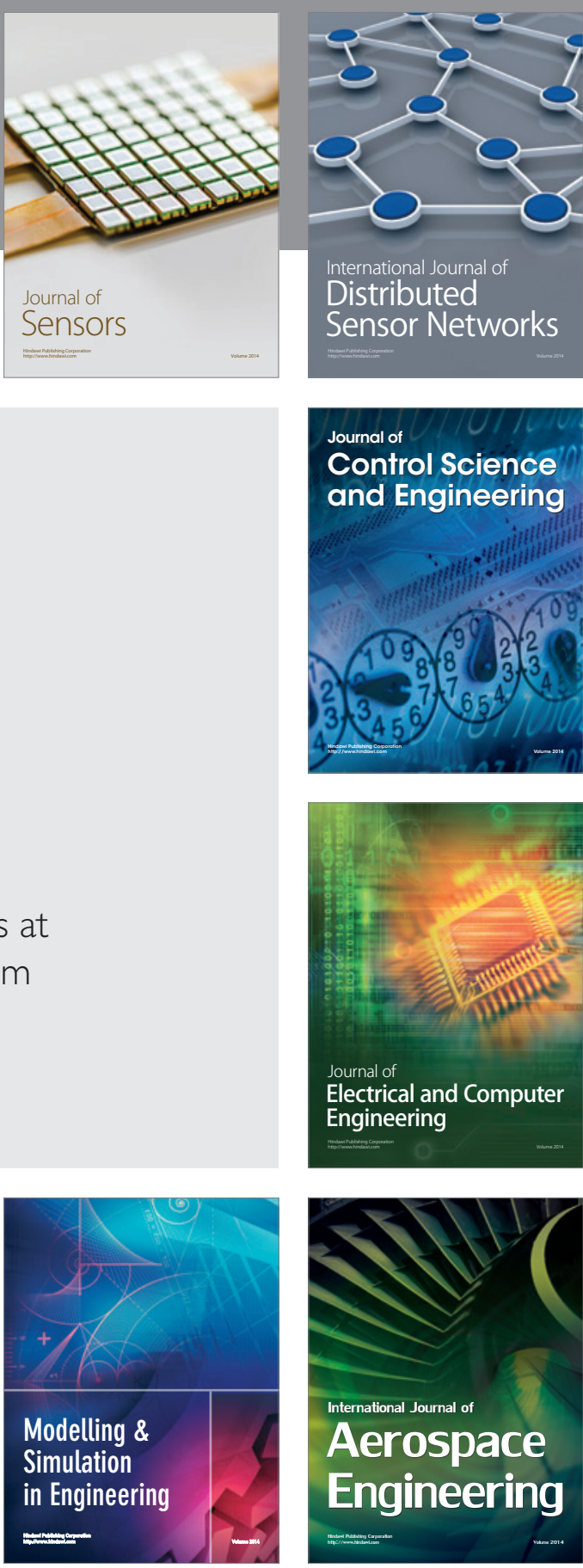

International Journal of

Distributed

Sensor Networks

$-$

Joumal of

Control Science

and Engineering
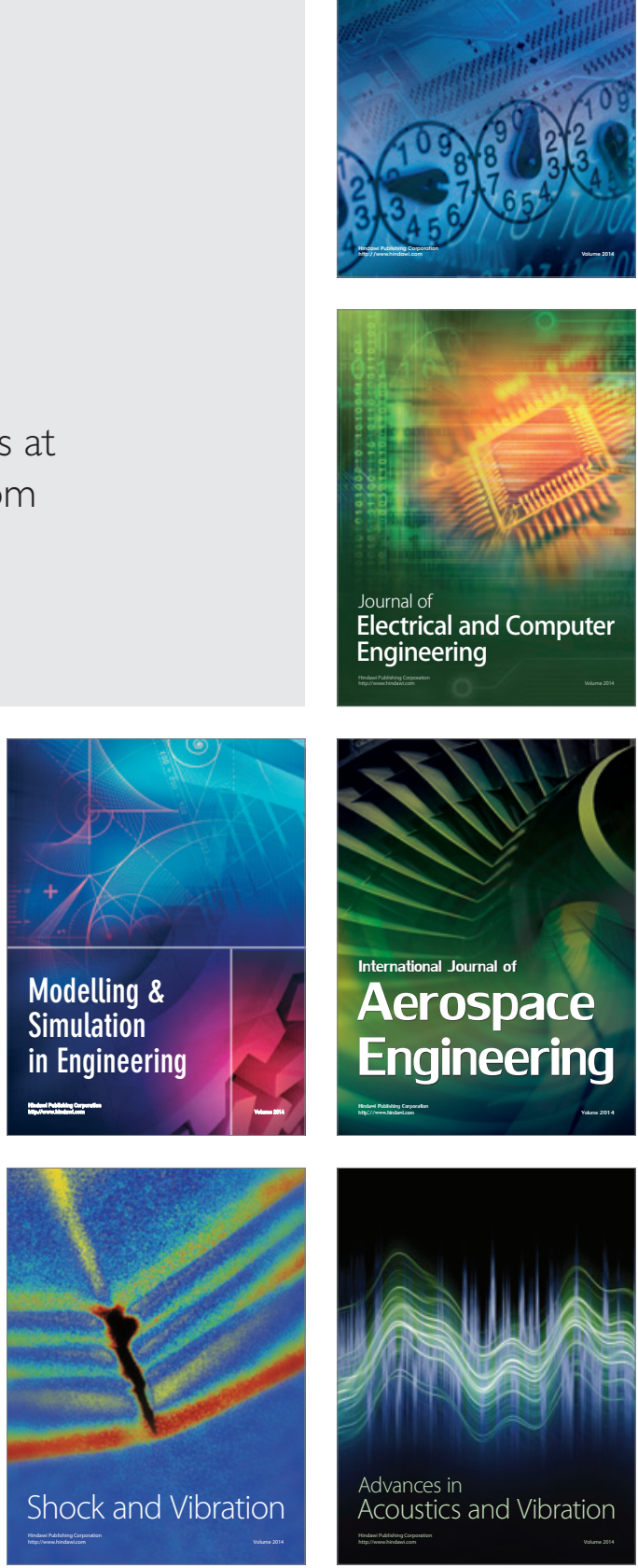